\title{
Resource allocation for energy efficient cellular systems
}

\author{
Stefan Videv*, John S Thompson, Harald Haas and Peter M Grant
}

\begin{abstract}
The need for more throughput in wireless cellular networks has been increasing in recent years. It has led to an increase in operational costs due to higher energy use as operators deploy more cell sites or increase transmission power at existing ones to satisfy demand. Energy costs are a major expense, and reducing them is a priority. This article presents a scheduler which aims to solve the problem of energy efficient resource allocation in orthogonal frequency division multiple access (OFDMA) cellular systems. The suggested approach is to make the resource scheduling process also consider energy costs as well as allow it to manipulate these by exploiting time/frequency vs energy efficiency trade-offs that are present in the system. The energy efficient score-based scheduler (EESBS) is a novel scheduler which takes energy costs into account when allocating resource blocks (RBs) to users. This allows it to promote energy efficiency in the system alongside throughput and fairness maximization. One of the means it has to manipulate users' expended energy is the bandwidth expanded mode (BEM). BEM is a technique that allows the scheduler to decrease a user's energy consumption by allocating it more RBs and maintaining a constant data rate. This is possible when the energy consumption is dominated by the energy used for data communication as opposed to control channel overhead transmission. Time compression mode (TCOM) is a technique that is complementary to BEM. It allows for energy savings through a reduction of the number of allocated RBs to a user when the energy consumption is dominated by the transmission of signaling traffic. Both BEM and TCOM need to be employed by an energy-aware scheduler like EESBS in order to extract the maximum performance gains. A realistic framework modeling future cellular systems is established to test the performance of the proposed techniques. Within this framework, EESBS generates an average energy saving of 29\% over a frequency selective proportional fair (FsPF) benchmark. EESBS coupled with BEM or TCoM achieves a saving of 38\% over the same benchmark. These savings are achieved with no detriment to user satisfaction in terms of achieved data rate.
\end{abstract}

\section{Introduction}

Wireless cellular communications have seen rapid development in recent years. With the advent of the smart phone, the desire for higher data rates has grown rapidly. It is commonly accepted that the increase is exponential with time [1]. From an operator's point of view, this is not desirable, as revenue has been increasing at a linear rate at best. This has resulted in a drive to reduce the operational costs associated with wireless cellular networks. Base stations' (BSs) energy consumption is one of the main contributors to the aforementioned cost. There has not been any significant coordinated attempt to optimize BSs energy

\footnotetext{
* Correspondence: s.videv@ed.ac.uk

School of Engineering and Electronics, Joint Research Institute for Signal and Image Processing, The University of Edinburgh EH9 3JL, Edinburgh, UK
}

performance in the past. Also, heightened environmental awareness has emerged as an additional incentive to minimize operational impact. All these factors have led to several initiatives which target a reduction of the energy consumption of wireless cellular networks by between 10 to 100 times through future research-for example, the mobile Virtual Center of Excellence's Green Radio project [2], the EARTH project [3], Greenet [4], and recently GreenTouch.

As a result, research has emerged on the topic of energy-efficient scheduling. This topic has previously been discussed widely in wireless sensor networks $[5,6]$. However, most of the work is in the context of relaxing quality of service constraints for energy efficiency. Within the traditional multi cell literature, the main topic of research is achieving higher spectral efficiency 
with an additional emphasis on fairness among users [7]. The focus is moving to increasing the energy efficiency of BSs towards that of mobile terminals. Han et al. [8] compare several schedulers in terms of throughput, fairness and energy efficiency. Their proposed fair cluster algorithm is focused on providing fairness over the user population but is also able to deliver energy efficiency improvements of about $12 \%$ compared to a round robin (RR) benchmark. The other proposed greedy algorithm, provides a 10 -fold reduction in used energy, unfortunately at the expense of a detrimental reduction in both throughput and power fairness as measured by an adaptation of Jain's fairness index. Yao and Giannakis [6] have proposed an energy efficient scheduling algorithm based on non-uniform time division multiple access (TDMA) that varies transmission length based on channel state and the size of the transmitted information. Their scheduler is able to achieve a reduction in used energy of up to $80 \%$ at the cost of longer transmission times. Interference mitigation is another of the key means to improve energy efficiency. Recently, interference mitigation techniques have been adapted to work in systems that employ multiple-input and multiple-output (MIMO) transmissions [9,10]. Another important topic is sleep modes [11-13]. These traditionally allow the system to actively alter its energy consumption by turning on and off its radio frequency (RF) front end depending on traffic conditions and transmission requests. Recent work by Wang et al. [14] focuses on pooling together under-loaded time slots (TSs), so that unused ones can be switched off. This allows for an interactive sleep mode that is able to respond to network and user demands in a better way. Significant gains of up to $90 \%$ are achieved for very low load factors. Gains are possible as long as the load factor is less than $60 \%$. As femto-cells are slowly being rolled out, research into optimum BS deployment is moving in the direction of optimizing for energy efficiency and not only coverage and delivered throughput [15]. However, the gains from deploying femto cells are strongly dependent on the particular scenario of use.

This article presents a novel scheduler, the energy efficient score-based scheduler (EESBS), and two novel techniques that are able to manipulate energy expenditure through resource scheduling, bandwidth expansion mode (BEM) and time compression mode (TCoM), that work together to reduce energy consumption in a wireless cellular system. The novelty of the three techniques lies in the scheduler being energy aware, and having the ability to use BEM or TCoM when it is beneficial to the system in terms of energy consumption. This is done without loss in other performance parameters.

The rest of the article is organized as follows. Section 2 introduces EESBS. This is followed by a short overview of BEM in Section 3, and theoretical derivations on its performance. Next TCoM is introduced along with a derivation of its performance. Simulation set-up and empirical results are presented in Section 5. The article concludes with Section 6.

\section{Energy efficient scheduling}

A major component of any energy efficient wireless communication system is the resource scheduler. A well designed system needs to be aware of the energy costs that different resource allocation decisions entail. This awareness allows the system to integrate any performance enhancements simply as alternative allocation options available to reduce energy consumption while satisfying users' demands.

\subsection{Score based scheduling}

The concept of a score based scheduler is proposed in [16]. In a TDMA-like system, a user $i(t)$ with the best score is selected at slot $t$ to transmit:

$$
i(t)=\underset{j=1, \ldots, U}{\operatorname{argmin}} s_{j}(t),
$$

where $U$ is the total number of users in the system, $s_{j}$ $(t)$ is the score of user $j$ at slot $t$, which corresponds to the rank of his current transmission rate $r_{j}(t)$ within the already observed values $r_{j}(t), r_{j}(t-1), \ldots, r_{j}(t-W+1)$ with $W$ being the window size in time. When there are two users with the same score, one is chosen at random. Formally, the scores are calculated as follows:

$$
s_{i}(t)=1+\sum_{l=1}^{w-1} \mathbb{1}_{\left\{r_{i}(t)<r_{i}(t-1)\right\}}+\sum_{l=1}^{w-1} \mathbb{1}_{\left\{r_{i}(t)=r_{i}(t-1)\right\}} X_{l},
$$

where $\mathbb{1}$ is the indicator function which returns 1 if the condition in the brackets is true and 0 otherwise and $X_{l}$ 's are independent identically distributed random variables that take on value from $\{0,1\}$ with $\operatorname{Pr}\left(X_{l}=0\right)$ $=1 / 2$.

Score based scheduling evaluates each user's performance on a future TS relative to their past experience, and assigns the aforementioned TS to the user who can benefit the most relative to their history.

\subsection{Energy efficient score based scheduler (EESBS)}

The score based scheduler in its basic form outlined above cannot be used to enhance energy efficiency while not compromising fairness and delivered data rate. The metric used for rating the resources needs to be changed, as well as the whole approach being adapted to OFDMA rather than TDMA systems. Resource allocation needs to be done and updated at a regular period to keep up with the channel conditions-for example every long term evolution (LTE) sub frame. In general, 
the shorter the time span for which resources are allocated, the better the energy efficiency would be. This is due to less time being allowed for the channel conditions to change.

To promote energy efficiency, RBs that can be used in a highly energy efficient manner need to be allocated first. The use of a metric for rating them that measures the energy efficiency of the RB can achieve the desired result. A large number of metrics can be used-total energy used, energy per bit delivered etc. In this work, total RF transmission power is used. A RB's energy performance is compared to that of the remaining $\mathrm{RBs}$ available for allocation to that user, and scores are assigned. Moreover, when a conflict between users arises-the user who can use the RB most efficiently among the contending users is allowed to transmit using it. The rest of the users are allocated their next best RB.

Also, fairness among users has to be assured while satisfying each user's data rate requirement as best as possible. To ensure that, users are allocated one RB at a time until their service requirements are met. By doing so, the effect that high demand users have on low demand ones is minimized. The total number of users in the system becomes more important rather than the existence of high demand users assuming all users are given the same priority. Please note the distinction between high priority and high demand users. High priority users will always have a detrimental effect on fairness in the system.

The equation for calculating scores hence becomes:

$$
s_{q}^{j}(t)=1+\sum_{k=1, k \neq q}^{M} \mathbb{1}_{E_{k}^{j}(t)>E_{q}^{j}(t)}+f^{j}\left(m_{j}\right),
$$

where $s_{q}^{j}(t)$ is the score for RB $q$ for user $j$ at TS $t$, $E_{k}^{j}(t)$ is the energy metric evaluated for user $j$ on RB $k$ at $t, M$ is the total number of $\mathrm{RBs}$ and $\dot{f}\left(m_{j}\right)$ is a penalty function based on the number of already allocated RBs for the user. Lower scores indicate more desirable RBs. The energy metric and penalty function used within this work are defined as $E_{k}^{j}(t)=P_{k}^{j}(t)$, where $P_{k}^{j}(t)$ is the RF transmission power required on RB $k$ for user $j$, and $f$ $\left(m_{j}\right)=m_{j}$, respectively. It is in theory possible to assign each user a separate penalty function, as well as to have a penalty function that prioritizes users based on different criteria, such as vulnerability, subscription plan etc. This makes EESBS a versatile and easy to tailor scheduling technique.

A pseudo code implementation of the score based scheduler can be found in Algorithm 1. The RESOLVE clause represents the process through which conflicts between users are resolved. A conflicting RB is allocated to the user who needs the least amount of energy to use it, whereas the rest of the users are allocated their next best RB according to the calculated scores.

Algorithm 1. Amended score-based scheduler

INITIALIZE requiredResouces $(1 \ldots j) \leftarrow$ calculate number of required RBs for all users

while sum(requiredResources) $>0$ do

CALCULATE $s_{q}^{j}(t)=1+\sum_{k=1, k \neq q}^{M} \mathbb{1}_{E_{k}^{j}}(t)>E_{q}^{j}(t)+f^{j}\left(m_{j}\right)$

for all users and RBs

for $\mathrm{i}=1$ to number of BSs do

FIND best RB for each user connected to this BS based on $s_{q}^{j}(t)$

if User's best RB is not allocated AND is usable

AND is not another user's best RB

then

ALLOCATE RB to user end if

if There were conflicting RBs between users then

RESOLVE conflicts by allocating RB to most efficient or priority user

ALLOCATE next best RBs, based on $s_{q}^{j}(t)$, to

the remaining users

end if

end for

if There are no available RBs for allocation then EXIT while loop

end if

end while

RUN power control algorithm

The last step in the scheduling algorithm is to run a power control subroutine. Power control is necessary to ensure that the minimum feasible transmission powers can be calculated, which allows the proposed techniques to be correctly evaluated. This ensures the most efficient operation of the system. The algorithm of choice here is the Foschini-Miljanic [17] power control algorithm due to its simplicity and low computational overhead. It is based on the following iterative control equation:

$$
P_{q}^{j}(t)=\frac{\Gamma_{q}}{\gamma_{q}^{j}(t-1)} P_{q}^{j}(t-1),
$$

where $P_{q}^{j}(t)$ is the transmission power used for user $j$ on $\mathrm{RB} q$ for TS $t, \Gamma_{q}$ is the SINR target for $\mathrm{RB} q$, and $\gamma_{q}^{k}(t-1)$ is the achieved SINR at the time instance prior to allocating resources. The power control subroutine should be allowed to run until the difference in the transmission power vectors between iterations, $\varepsilon$, has converged. Within this work, $\varepsilon$ is defined to be $1 \%$ of the maximum BS RF transmission power. This is reflected in a relatively low number of control loop iterations required-on average 6-7. The transmission 
powers used to calculate the energy efficiency metric used for allocation will be different from the ones actually used for transmission. This will result in a difference in the energy efficiency expected at allocation and the achieved one. Since the problem of energy efficient allocation of RBs and power to transmit on them is NP hard, this article presents a heuristic solution. Within this framework, each allocation iteration improves on the energy efficiency of the system.

\section{Bandwidth expansion mode}

\subsection{General description}

There are times in the operation of any cellular wireless network when there are only a few users who may demand high transmission rates-for example early in the morning or in rural area cell sites. It is reasonable to assume that at those times unused bandwidth should be available in cells operated under a LTE system. It is hence advantageous to employ techniques/algorithms that trade-off unused bandwidth for energy efficiency.

BEM [18] operates by allowing a user to expand his limited bandwidth by an integer factor of $\alpha$ while preserving the already achieved data rate. The allocated bandwidth is increased while the modulation order and signal-to-interference-plus-noise-ratio (SINR) requirement per frequency channel, $\Gamma_{q}$, are decreased to maintain the communication rate. The technique exploits the energy saving opportunity created through the fact that the transmission rate scales logarithmically with the achieved SINR, and linearly with the used bandwidth or number of channels. Hence, an energy saving can be incurred in a communications system without any degradation in service. Moreover, robustness of communication is increased as data is transmitted over more channels or RBs.

Consider the following example frequency RB allocation. A user is allocated 4 out of a maximum $10 \mathrm{RBs}$ and transmits on them with relatively high power. This presents the opportunity to allocate an additional 4 RBs, and switch to a lower order modulation scheme if possible. An overall reduction in expended energy can be hence achieved. For example, doubling the used bandwidth and going from 16-QAM to 4-QAM increases the energy consumption by a factor of two, but at the same time reduces it by 3.16 times due to the more efficient modulation which results in a net reduction of 1.58 times. In a more general scenario, a user can expand his bandwidth footprint by a factor of $\alpha$ that is a real number by choosing an appropriate modulation scheme, SINR target, coding scheme and other required link parameters. However, it must be stressed that only an integer number of RBs can be allocated to each user. The integer constraint on $\alpha$ is imposed within this work for simplicity.
The reduction in energy due to BEM can be calculated in the form of energy consumption gain (ECG) as:

$$
\mathrm{ECG}_{\mathrm{BEM}}^{\mathrm{RF}}=\frac{E_{\text {ref }}}{E_{\text {new }}}=\frac{\Gamma_{q}}{\alpha\left(\sqrt[\alpha]{1+\Gamma_{q}}-1\right)},
$$

where $E_{\text {new }}$ is the energy required for operation by the investigated system, $E_{\text {ref }}$ the energy for the reference system, $\alpha$ is the bandwidth expansion factor, and $\Gamma_{q}$ is the required SINR on the link prior to expanding the bandwidth. Also, the RF superscript stands for radio frequency, as the expression above takes into account only the radiated RF energy used for data transmission. The expression is derived starting from Shannon's capacity equation-the rate for the system with BEM is equated to that to the reference system without BEM, and the BEM SINR target is computed as a function of the original one. The energy consumption of BEM and the reference system are then calculated to arrive at the ECG expression.

\subsection{Effect of control channel overhead}

Allocating additional resources raises the question of what happens to the control channel overhead. This needs to be answered in order to correctly assess the gains from BEM. Hence, the behavior of control overhead transmissions needs to be modeled and included in the analysis. Typically, cellular systems have a broadcast channel which carries information that allows the other channels in the system to be configured and used for communication. The 3rd Generation Partnership Project (3GPP) LTE makes use of a Physical Broadcast Channel $(\mathrm{PBCH})$ which carries a 'Master Information Block' (MIB), as well as a Physical Downlink Control Channel $(\mathrm{PDCCH})$. Both are a part of the Physical Downlink Shared Channel (PDSCH)-they are allocated particular resources in time and frequency [19].

We assume that the $\mathrm{PBCH}$, reference, and synchronization signals are transmitted with a constant power, so that they can achieve coverage of the complete intended cell site. $\mathrm{PBCH}$ information is transmitted every $40 \mathrm{~ms}$ or every 40 sub-frames. In addition to that, in every sub-frame there is PDCCH data as well as reference signals that are transmitted. The PDCCH data can be configured to occupy the first 1,2 , or 3 OFDM symbols.

If we are to quantify the total overhead due to $\mathrm{PBCH}$ and PDCCH using the values presented above, we calculate that it varies between approximately 12.9 and $26.9 \%$. It is important to note that this is overhead both in terms of communication time and energy. The contribution of $\mathrm{PBCH}$ is relatively minor-approximately $1.3 \%$ relative to the complete system. Of course, there are also other types of overhead that are not considered here-for example Physical Control Format Indicator 
Channel (PCFICH) and Physical Hybrid ARQ Indicator Channel (PHICH). These do not constitute a significant part of the control channel overhead in the system. It is also important to note that the PDCCH could be turned off if the particular frequency resource blocks (RBs) are not going to be used, whereas the $\mathrm{PBCH}$ cannot be turned off as it is required so that new users can connect to the network. Hence, only the overhead from the $\mathrm{PDCCH}$ will be considered in this article.

The following assumptions are made throughout this work. The PDCCH along with the reference signals can be turned off if the particular RB is not in use until the next allocation cycle. This means that the control channel overhead due to PDCCH can be modeled as a transmission that occupies a fraction of the TS at a constant pre-set RF power.

Allocating more resources to users by allowing them to enter BEM means that there will be an additional control channel overhead associated with these which will affect the overall energy use. It is important to model this so that the real-world performance of this technique can be better assessed.

The ECG expression for BEM needs a significant alteration to account for the effect of a constant power control transmission. After simplification, the new ECG expression for transmitter $k$ and intended receiver $j$ becomes:

$$
\mathrm{ECG}_{\mathrm{BEM}}^{\mathrm{CC}}=\frac{\sum_{q=1}^{n}(1-\phi) \frac{\Gamma_{q}\left(N+I_{q}\right)}{G_{k j}^{q}}+\phi P_{\mathrm{ref}}}{\sum_{q=1}^{\alpha n}(1-\phi) \frac{\left(\sqrt[\alpha]{1+\Gamma_{q}}-1\right)\left(N+I_{q}\right)}{G_{k j}^{q}}+\phi P_{\mathrm{ref}}}
$$

where $n$ is the total number of RBs allocated, $N$ is the noise floor, $I_{q}$ is the interference received on $\mathrm{RB} q, G_{k j}^{q}$ is the path gain for $\mathrm{RB} q, \phi$ is the fractional control channel overhead, and $P_{\text {ref }}$ is the power with which the reference signals are transmitted.

The new Equation, (6), is plotted for an example single carrier system with $\alpha=2$ and $\phi=0.15$ in Figure 1 where RF output power refers to the power radiated from a BS antenna in the system.

Control channel overhead introduces new patterns in the gain behavior for BEM. There is a variation in the gain experienced depending on the ratio between the data and the control channel transmission power. The higher the ratio, the closer to the previously calculated theoretical gains the system performs as the effect of

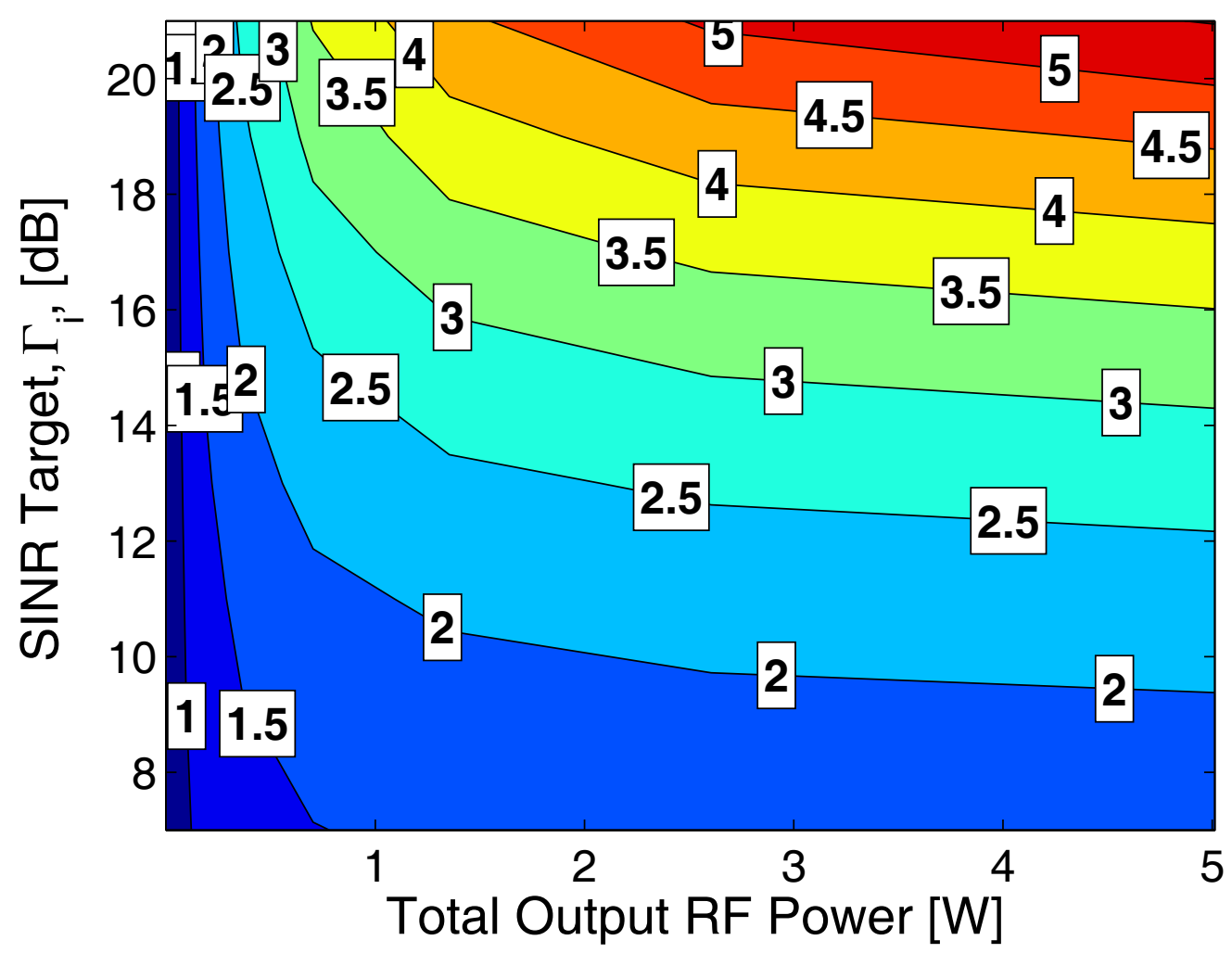

Figure 1 ECG for BEM with control channel overhead ( $\phi=0.15$ and $\alpha=2$ ). 
control transmission overhead becomes negligible. For low values of this ratio, the energy consumption of a communication node is dominated by the control channel overhead. In that case, increasing the number of RBs that are in use compromises the energy efficiency making the use of BEM not desirable. Having a higher initial SINR target before employing BEM results in larger gains from the technique as already established.

\subsection{Effect of hardware operational efficiency}

Switching the operation of a BS from one output RF power level to a lower one has an effect on the efficiency with which RF power is delivered. It is widely accepted that the farther away from peak RF output a BS is operated, the worse its efficiency is. Hence, in the context of this investigation, it is important to use an accurate BS energy consumption model.

Three versions of the same model are used throughout this article. The BS power consumption model is the one adopted in [20]. It is governed by the following equation:

$$
P_{\mathrm{BS}, \text { in }}=P_{\mathrm{BS}, 0}+\Delta_{P} P_{\mathrm{BS}, \text { out }}
$$

where $P_{\mathrm{BS} \text {,in }}$ is the required power drawn by the $\mathrm{BS}$ in Watts, $P_{\mathrm{BS}, 0}$ is the idle power consumption i.e. when no RF power is used for data transmission, also in Watts, $\Delta_{P}$ is a scaling parameter, and $P_{\mathrm{BS} \text {,out }}$ is the required output RF power in Watts. The maximum value allowed within this model for $P_{\mathrm{BS} \text {,out }}$ is $P_{\mathrm{BS} \text {,out }}^{\max }$ which would generally be a design parameter of the $\mathrm{BS}$. The appropriate parameters for a macro BS are $P_{\mathrm{BS}, 0}=712 \mathrm{~W}, \Delta_{P}=$ 14.5 , and $P_{\mathrm{BS}, \text { out }}^{\max }=40 W[20]$. A plot of the required input power over the range of operation can be found in Figure 2. This model reflects the current state of BSs.

It is expected that the efficiency of BS hardware will improve in the near future. Also, input power requirements will scale much better with the required output $\mathrm{RF}$ power as power amplifier (PA) efficiency is improved and the overall BS consumption reduced [2]. Both these advancements should add to the benefits obtained from BEM and TCoM. The two techniques manipulate the dynamic component of the energy drawn during operation. It is expected that as BSs efficiency is improved, this component will become increasingly dominant over the quiescent drain hence increasing the benefit from BEM and TCoM. This is why two additional

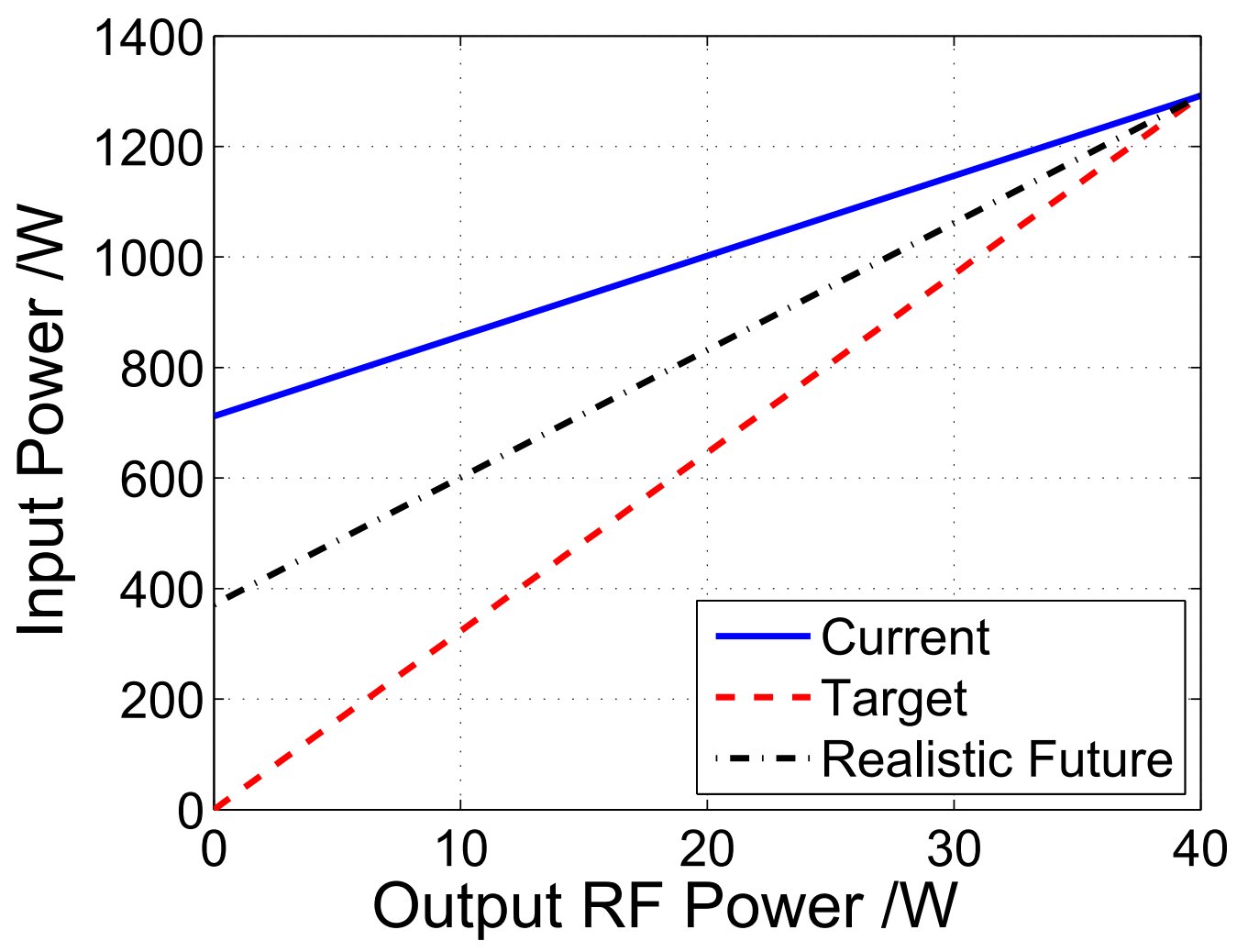

Figure 2 BS model. 
consumption models are proposed and adopted. One models the desired ideal performance of the future BS, and the other represents a more realistic proposition. Both can be expressed in the same manner as (7). The parameters for the realistic model are $P_{\mathrm{BS}, 0}=371 \mathrm{~W}, \Delta_{P}$ $=23.025$, and for the idealistic one $-P_{\mathrm{BS}, 0}=0 \mathrm{~W}, \Delta_{P}=$ 32.3 .

The first, pessimistic, model is used for evaluating the techniques theoretically. This gives a more realistic view on the gains as the theoretical results make use of idealistic assumptions leading to high predicted gains. However, all three models are used to evaluate the energy savings through simulation in Section 5.

Keeping in mind the operation of BEM, the already calculated ECG can be modified to account for hardware operation at a given RF output as follows:

$$
\mathrm{ECG}_{\mathrm{BEM}}^{\mathrm{TOT}}=\frac{P_{\mathrm{BS}, \text { in }}\left(P_{\mathrm{BS}, \text { out }}\right)}{P_{\mathrm{BS}, \text { in }}\left(\frac{P_{\mathrm{BS}, \text { out }}}{\mathrm{ECG}_{\mathrm{BEM}}^{\mathrm{CC}}}\right)} \text {. }
$$

It is important to note that the above equation has a significant drawback. It does not account for the fact that any initial decrease or increase in interference due to one cell, and hence used energy, is reinforced by an additional one due to the decrease or respectively increase in interference in all of its neighbors. This is a dynamic effect akin to a cocktail party effect which is difficult to model theoretically.

A plot of (8) for a pre-set ratio of $P_{\text {data }} / P_{\text {ref }}=3.4, \alpha=$ 2 and $\phi=0.25$ can be found in Figure 3. This parameter combination is conservative-low transmission power and high overhead define a scenario where BEM should demonstrate comparatively modest gains. However, even in those conditions the technique provides significant gains. There are two trends to be observed in the figure. First, for a given $\Gamma_{q}$, the gain increases as the total RF output power increases. This means BEM is more useful in bad channel conditions. It also reveals that the variable energy efficiency of the BS which depends on the output RF load has a role to play in determining the overall gain from BEM. Second, for a given output RF power, the gain increases as $\Gamma_{q}$ increases. Higher $\Gamma_{q} \mathrm{~S}$ represent less energy efficient modulation schemes against which BEM is able to achieve higher gains.

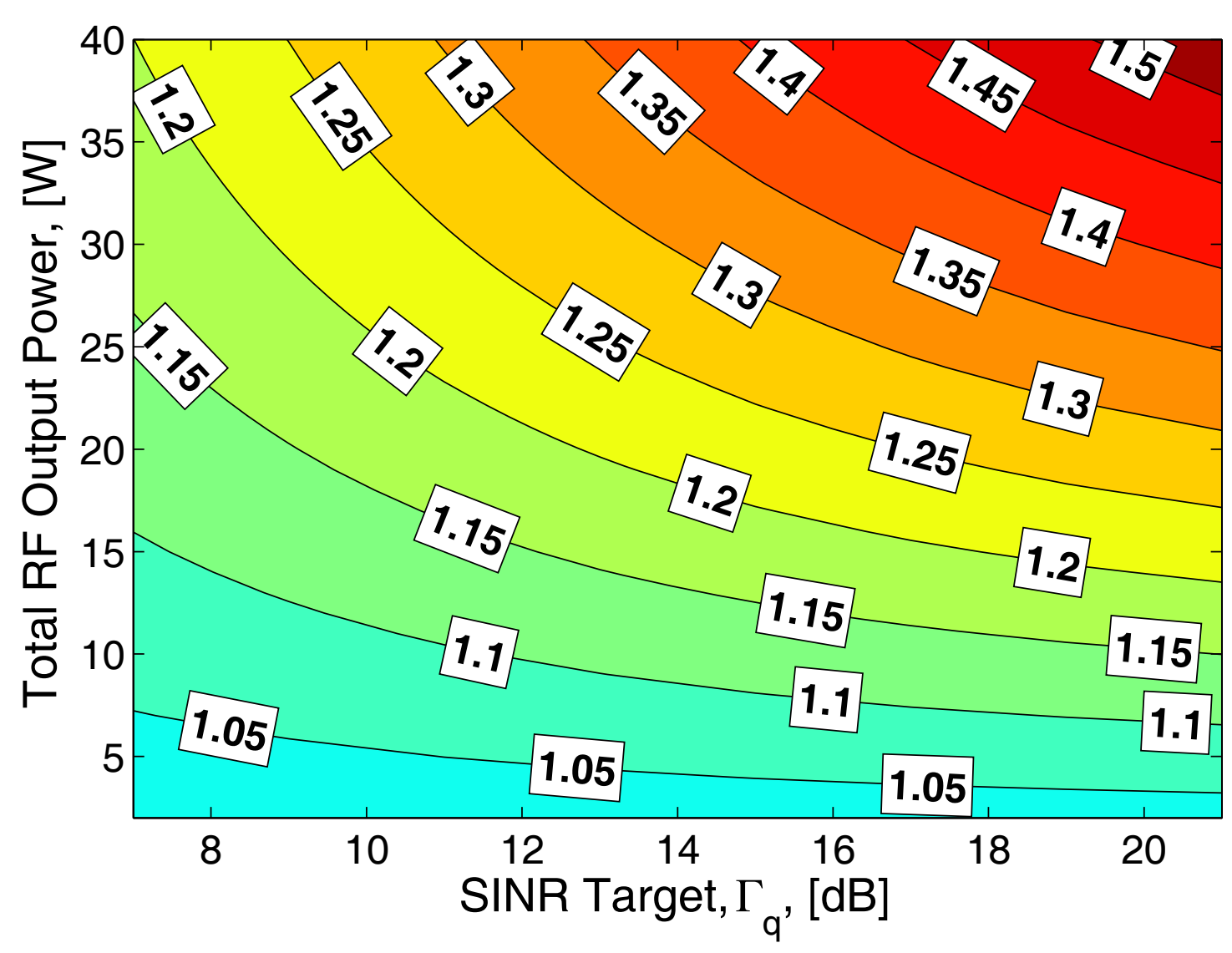

Figure 3 Overall ECG for BEM with fixed data transmission power $\left(P_{\text {data }} / P_{\text {ref }}=3.4, P_{\text {data }}=1.53 \mathrm{~W}, \alpha=2\right.$ and $\left.\phi=0.25\right)$. 
Figure 4 has been realized for a pre-set $\Gamma_{q}=11 \mathrm{~dB}$ and $P_{\text {ref }}=0.4 \mathrm{~W}$. The parameters are chosen to show that even in high overhead conditions BEM can deliver gains. As the transmission power per RB is increased the gain from BEM increases initially until it converges. This is due to the shift in ratio between overhead and data energy. A low data-overhead ratio means there will not be any gains from BEM. Conversely, a high dataoverhead ratio means there are high gains achievable. After the ratio becomes high enough, the achievable gain is no longer affected by overhead as it becomes insignificant.

\section{Time compression mode}

As shown in the previous section, BEM leads to good results when a user's energy consumption is dominated by energy used for data transmission and not control overhead communication. This means a part of the users cannot benefit from the use of BEM. A straightforward way to reduce the energy consumption of these users is to propose a complementary transmission mode. A similar idea has already been described in [14] for a time based implementation. In the prior work, underutilized RBs are lumped together in time without any change in the used modulation. RBs that are then not needed are turned off to conserve energy that would otherwise be wasted in control channel transmissions. In this work, RBs that are fully utilized on a relatively low modulation order are lumped together in time, or alternatively in frequency, and a higher order modulation is used. Energy savings are accrued through the reduction in overhead signaling. Naturally, this is done when the channel conditions allow the use of higher order modulation. One of the goals of this article is to determine if the TCoM technique can provide energy saving gains when deployed in realistic scenarios and in what conditions.

A comparison of the three different systems discussed so far can be found in Figure 5.

Decreasing the number of allocated frequency channels in the TCoM system leads to a decrease in the amount of overhead transmissions required, which suggests that users whose energy expenditure is dominated by control channel transmissions will benefit.

\subsection{Time vs. frequency implementations}

To be able to assess the difference between time and frequency implementations of TCoM, knowledge on

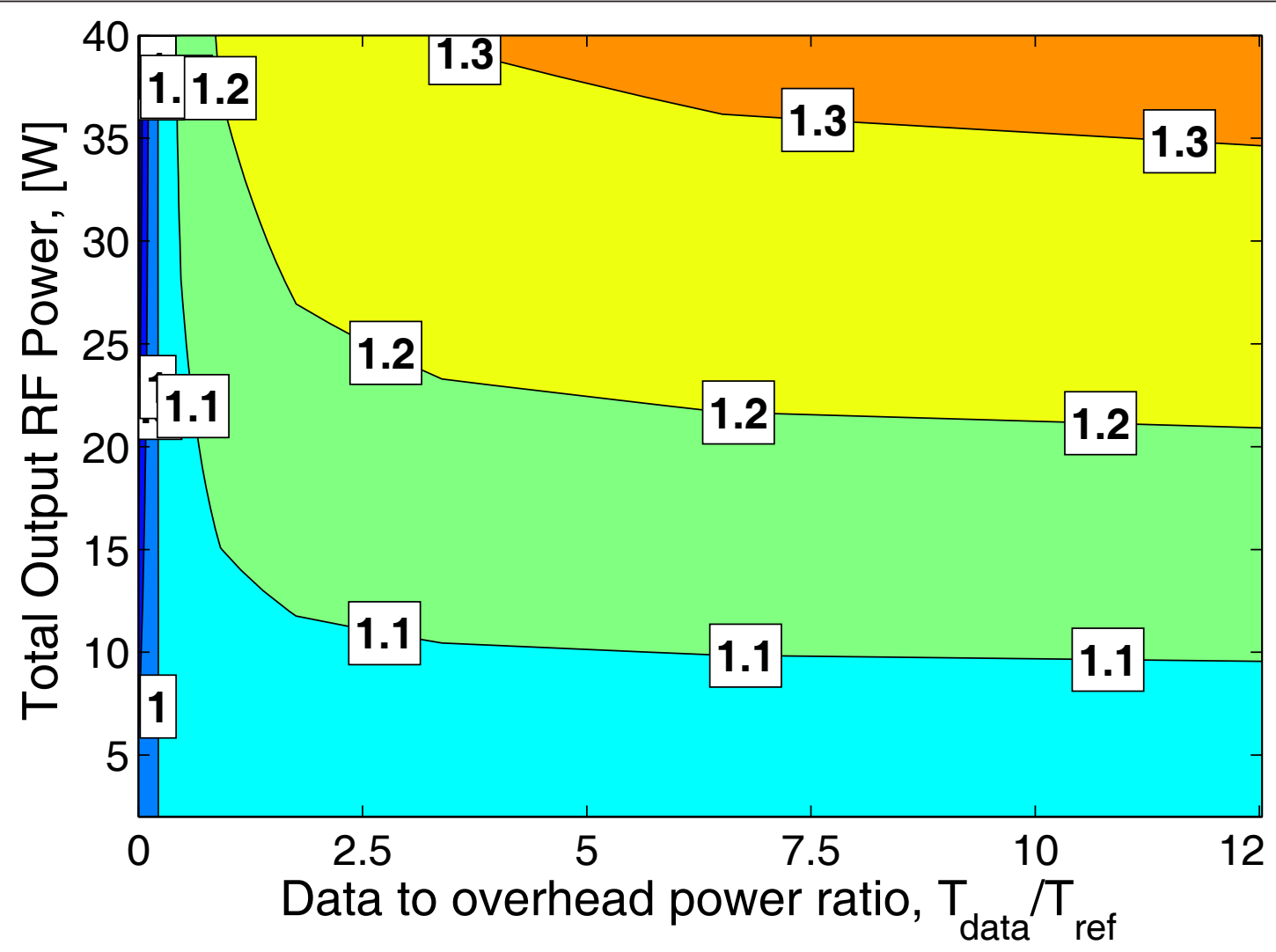

Figure 4 Overall ECG for BEM with fixed $\Gamma_{q}=11 \mathrm{~dB}(\alpha=2$ and $\phi=0.25)$. 


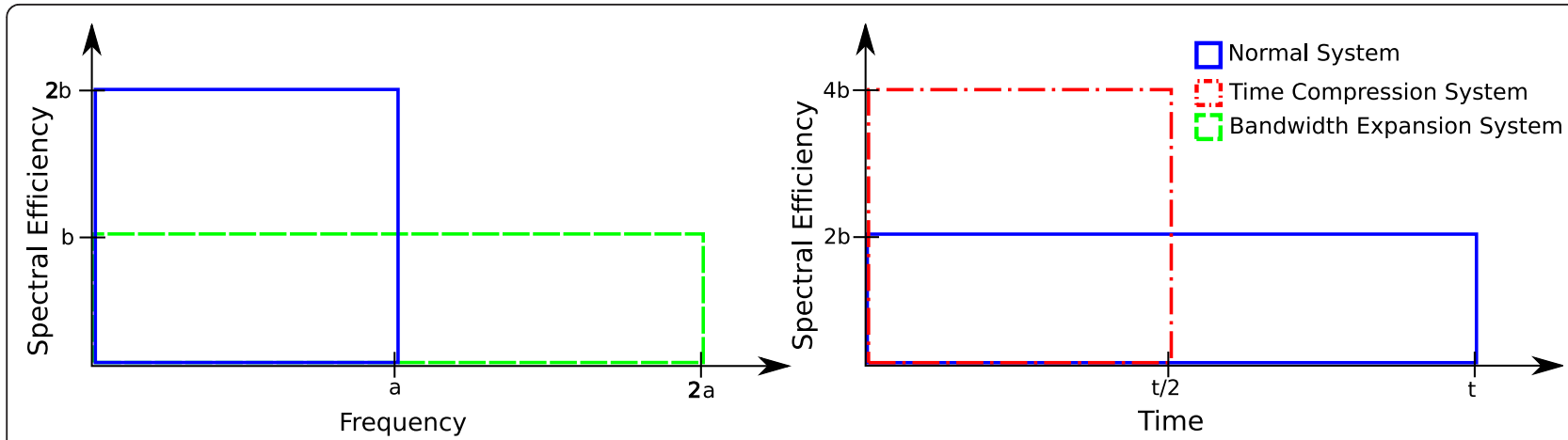

Figure 5 System descriptions-left: normal system vs BEM; right: normal system vs. TCoM.

how energy costs are generated in the system is required. In general:

$$
D=R L B,
$$

where $D$ is the delivered payload in bits, $R$ is the spectral efficiency in bits/s/Hz, $L$ is the length of transmission in seconds, and $B$ is the bandwidth in Hz. From this equation it is clear that by increasing the rate, it is possible to either decrease the time it takes for a transmission or decrease its bandwidth footprint. On the other hand, the used energy for a transmission can be calculated as:

$$
E_{\text {Used }}=P_{\text {data }} L_{\text {data }} n+P_{\text {ref }} L_{\text {ref }} n \text {, }
$$

where $E_{\mathrm{Used}}$ is the used total energy, $P_{\text {data }}$ is the power dedicated to data transmissions, and the overall transmission time $L=L_{\mathrm{data}}+L_{\mathrm{ref}}$ is split between user data and reference signaling. The above can be rewritten with the introduction of $\phi$ :

$$
E_{\text {Used }}=n L\left((1-\phi) P_{\text {data }}+\phi P_{\text {ref }}\right) .
$$

From the above, it emerges that a linear compression or expansion in either bandwidth or time of a transmission, while retaining the overall delivered payload, leads to the same change in used energy. For example, expanding the bandwidth by a factor of two, will yield the same result as taking twice as long to complete the transmission if we were to retain the size of the delivered payload and the used modulation scheme. In the case of reducing bandwidth or length of transmission, it should be possible to turn off the control channel transmissions on the unused RBs. The number of symbols used for a particular transmission of PDCCH is set by the BS. Explicitly, it is determined by the channel conditions to ensure that the transmission can be reliably decoded [19]. It should be possible to adapt this functionality in LTE, so that it supports the process of eliminating unnecessary overhead to increase energy efficiency.

\subsection{ECG derivation}

As outlined above, time and frequency implementations of TCoM should not differ in performance, hence only the frequency based system derivation of the gains is presented. To make sure that the two systems deliver the same amount of data, the following needs to be satisfied:

$$
R_{\mathrm{TCOM}}=R_{\text {benchmark }} \beta,
$$

where the subscripts denote the different systems and $\beta$ is the time/bandwidth compression factor, which is an integer similar to $\alpha$. From (12) and Shannon's capacity equation, we can derive the required SINR target for the TCoM system:

$$
\tilde{\Gamma}_{q}=\left(1+\Gamma_{q}\right)^{\beta}-1,
$$

where $\tilde{\Gamma}_{q}$ is the TCoM SINR target. From the basic SINR calculation formula, the required RF power can be calculated:

$$
P_{q}^{\tilde{\mathrm{RF}}}=\frac{N+I_{q}}{G_{k j}} \tilde{\Gamma}_{q} .
$$

The total expended RF energy to deliver a payload on $\beta$ RBs in the benchmark system and the same payload in the proposed system can be calculated as:

$$
\begin{aligned}
& E_{\text {benchmark }}=L \sum_{q=1}^{\beta}\left((1-\phi) P_{q}+\phi P_{\text {ref }}\right) \\
& E_{\mathrm{TCOM}}=L\left((1-\phi) \tilde{P}_{q}+\phi P_{\text {ref }}\right) .
\end{aligned}
$$

The RF ECG can now be calculated from basic principles and (14) as:

$$
\mathrm{ECG}_{\mathrm{TCOM}}^{\mathrm{RF}}=\frac{\beta \Gamma_{q}}{\left(1+\Gamma_{q}\right)^{\beta}-1},
$$


where the assumption is that all used RBs are experiencing nearly identical channel conditions. If the control channel transmissions are to be accounted for as before as well as different channel conditions, the above becomes:

$$
\mathrm{ECG}_{\mathrm{TCoM}}^{\mathrm{CC}}=\frac{\beta \Sigma_{q=1}^{n}(1-\phi) \frac{\Gamma_{q}\left(N+I_{q}\right)}{G_{\mathrm{kj}}^{q}}+\phi P_{\mathrm{ref}}}{\sum_{q=1}^{n}(1-\phi) \frac{\left(\left(1+\Gamma_{q}\right)^{\beta}-1\right)\left(N+I_{q}\right)}{G_{\mathrm{kj}}^{q}}+\phi P_{\mathrm{ref}}} .
$$

The above is plotted in Figure 6 for $\phi=0.25$ and $\beta=$ 2 to underline the gains from TCoM. The figure confirms that the users most likely to benefit from TCoM are ones that enjoy very good channel conditions while not requiring high data throughput, which means their energy consumption is dominated by control channel transmissions. Such users are most likely to be found in the cell center.

Following the manner of the BEM ECG derivation, (18) is modified to account for hardware efficiency:

$$
\mathrm{ECG}_{\mathrm{TCoM}}^{\mathrm{TOT}}=\frac{P_{\mathrm{BS}, \text { in }}\left(P_{\mathrm{BS}, \text { out }}\right)}{P_{\mathrm{BS}, \text { in }}\left(\frac{P_{\mathrm{BS}, \text { out }}}{E C G_{\mathrm{TCoM}}^{\mathrm{CC}}}\right)} .
$$

Figure 7 presents (19) plotted for a ratio of data to overhead transmission power, $P_{\text {data }} / P_{\text {ref }}$, of 0.0012 . This value is reasonable considering the path loss difference experienced by a user in the cell center and one on the cell edge. Two trends can be observed. First, the higher the total output RF power, the higher the gain from TCoM. This is due to the fact that the higher the initial loading the more efficient the operation of the system after the required total output power has been reduced through TCoM. Second, the higher the initial SINR target, the lower the gain from TCoM for a given total RF output level. This is due to the fact that more complex modulation techniques are less energy efficient than the simpler ones.

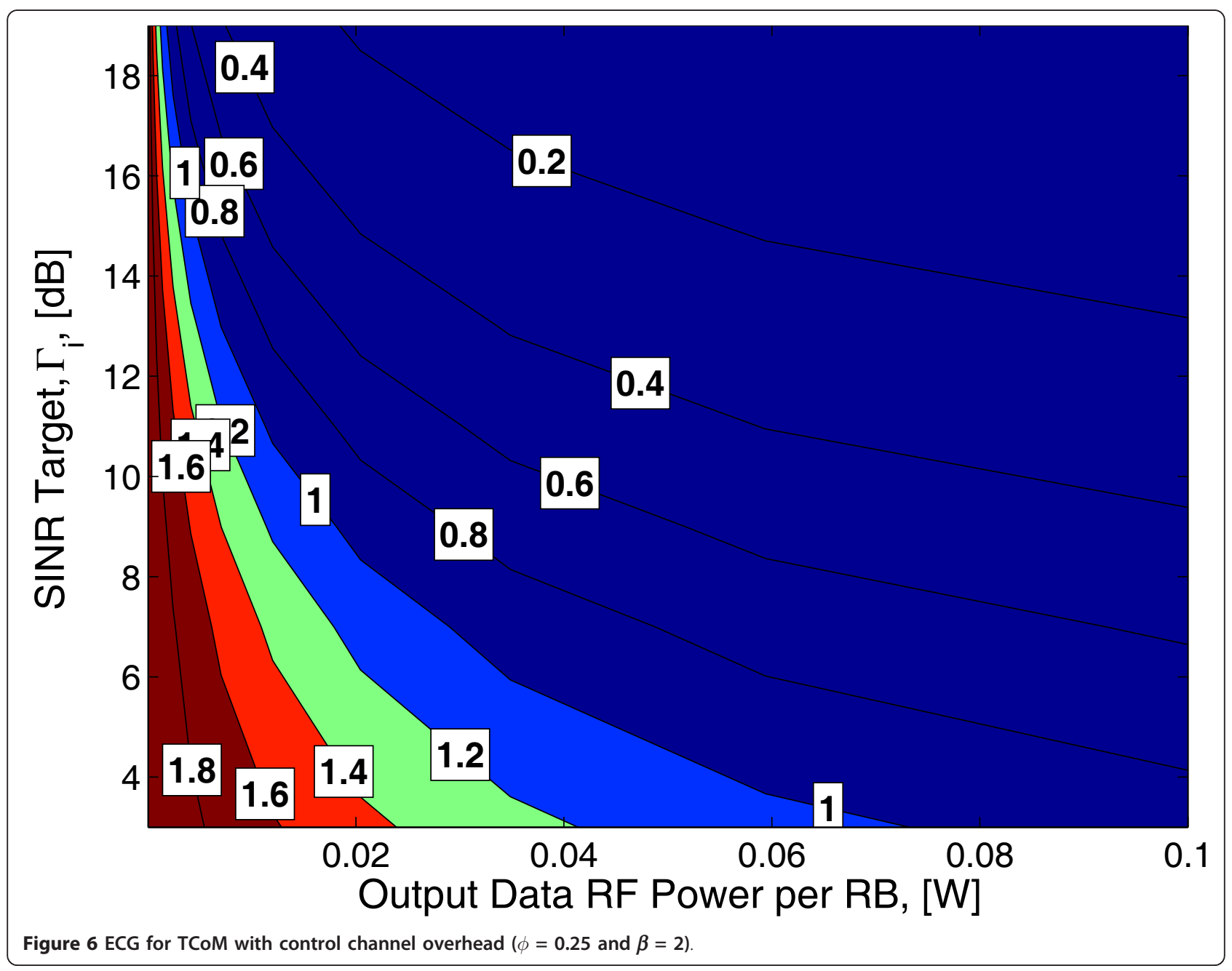




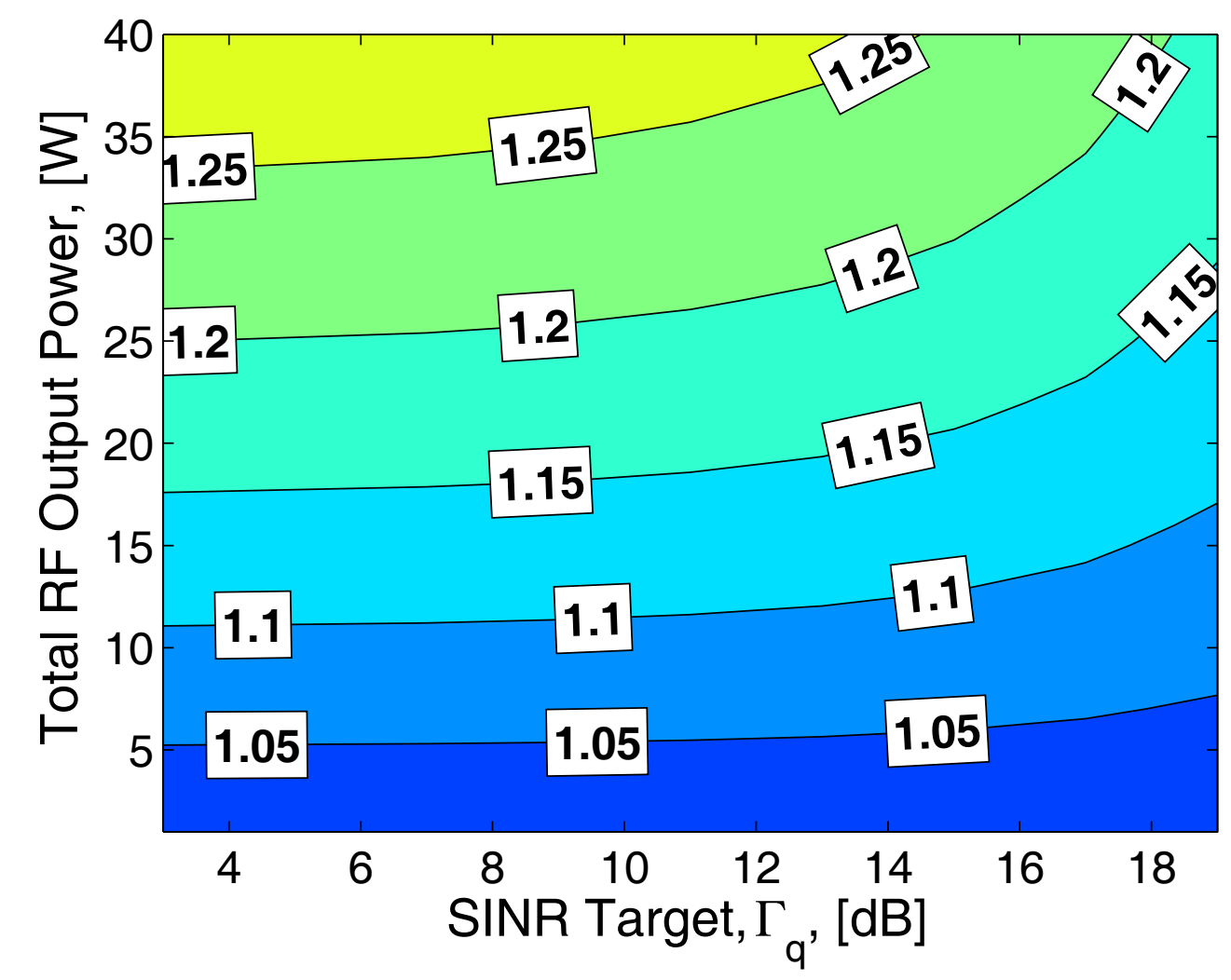

Figure 7 Overall ECG for TCoM with $\phi=0.25, \beta=2$, and $P_{\text {data }} / P_{\text {ref }}=0.0012$.

\section{Empirical results}

To validate the theoretical results, a simulation platform is used to evaluate the performance of EESBS and a system that combines EESBS, BEM, and TCoM, further referred to as the bandwidth scheduling system, against a frequency selective proportional fair (FsPF) scheduler as the one discussed in the Problem Formulation section of [21]. The overall system framework is modeled after LTE-it is an OFDMA based system with parameters taken from the LTE standard proposal documentation.

\subsection{Simulation set-up}

The simulation parameters employed can be found in Table 1 . The number of users per cell is picked from a uniform distribution defined between 5 and 9 users per BS. Each user is assigned a target data rate from a uniform distribution comprised of the following elements$(0.5,1,1.5,2)$ Mbps. Those result in a bandwidth loading between 20 and $50 \%$. The chosen scenario topology is a two-tiered wrap around a central cell. It is generally accepted that this scenario results in a realistic interference environment in the central cell. Performance data is collected only from this cell. However, all cells operate in the same manner. The channel model used is the
Urban Micro cell model (UMi) as defined in [22]. Frequency selective fading based on the clustered delay line model for scenario B1 is incorporated as described in

Table 1 System parameters

\begin{tabular}{ll}
\hline Parameter & Value \\
\hline Total bandwidth & $20 \mathrm{MHz}$ \\
Carrier frequency & $2.14 \mathrm{GHz}$ \\
Resource bandwidth & $180 \mathrm{kHz}$ \\
Number of RBs & 100 \\
Subcarriers per RB & 12 \\
BS maximum power & $46 \mathrm{dBm}$ \\
User speed & $3 \mathrm{~km} / \mathrm{h}$ \\
SINR targets, $\Gamma_{q}$ & $-6-20 \mathrm{~dB}$ \\
Data rates & $0.1523-5.5547 \mathrm{bits} / \mathrm{symbol}$ \\
Users per BS & $5-9$ \\
Required data rate per user & $0.5,1,1.5,2 \mathrm{Mbps}$ \\
Inter-site distance & $870 \mathrm{~m}$ \\
Overhead & $12.9 \%$ \\
Control channel SINR target, $\Gamma_{\text {ref }}$ & $3.4 \mathrm{~dB}$ \\
Antenna gain & $14 \mathrm{~dB}$ \\
Bandwidth expansion factor, $\alpha$ & 2 \\
Bandwidth compression factor, $\beta$ & 2 \\
\hline
\end{tabular}


[23]. A infinite size buffer model is used to ensure that all MSs want to transmit at all TSs.

Resource blocks that cannot be used for transmission due to experiencing temporary deep fades or high path loss are not considered during the process of allocation. BEM and TCoM require an $\mathrm{RB}$ allocation as a starting point to their operation. The allocation that is achieved by EESBS is used for that purpose. BEM and TCoM then provide iterative improvements over the starting allocation.

The FsPF scheduler operates by applying the proportional fair principle to each RB at a time, and allocating each $\mathrm{RB}$ to the user who maximizes the fairness ratio. To avoid initial allocation conflicts, the order in which RBs are considered within each BS is randomized.

The user specific penalty function used within the EESBS scheduler is $f^{k}\left(m_{k}\right)=m_{k}$, where $m_{k}$ is the number of currently allocated RBs to user $k$. This means that the more RBs are allocated to a user, the larger the penalty resulting in a fairer allocation. The scheduler also chooses whether to allocate resources based on their effect on the overall cell energy efficiency in BEM and TCoM. If the allocation of a particular RB affects negatively the predicted overall efficiency of the BS, it is not allocated. The current resource allocation and channel conditions are used to estimate the required energy.

Also, the control channel transmission power, $P_{\text {ref }}$, has to be defined in a meaningful manner. Control channel transmissions need to be able to reliably reach any user within the radius of the BS. Hence, $P_{\text {ref }}$ needs to be defined with respect to the cell radius and an SINR target, $\Gamma_{\text {ref, }}$ that defines the modulation used for control channel communication. The following equation is used to calculate the required control channel transmission power per RB:

$$
P_{\text {ref }}=\frac{\Gamma_{\text {ref }}\left(N+3.7710^{-14}\right)}{G(d)},
$$

where $G(d)$ is the worst path gain for a distance of $d$ between the user and BS including log-normal shadowing up to two sigma away from the mean as that covers $95 \%$ of the user population. The value used for the interference component that is to be tolerated is the maximum possible for the given scenario at the cell edge. The data transmission SINR targets are chosen using Shannon's channel capacity equation by adding an additional $3 \mathrm{~dB}$ to the calculated SINR targets. This is a

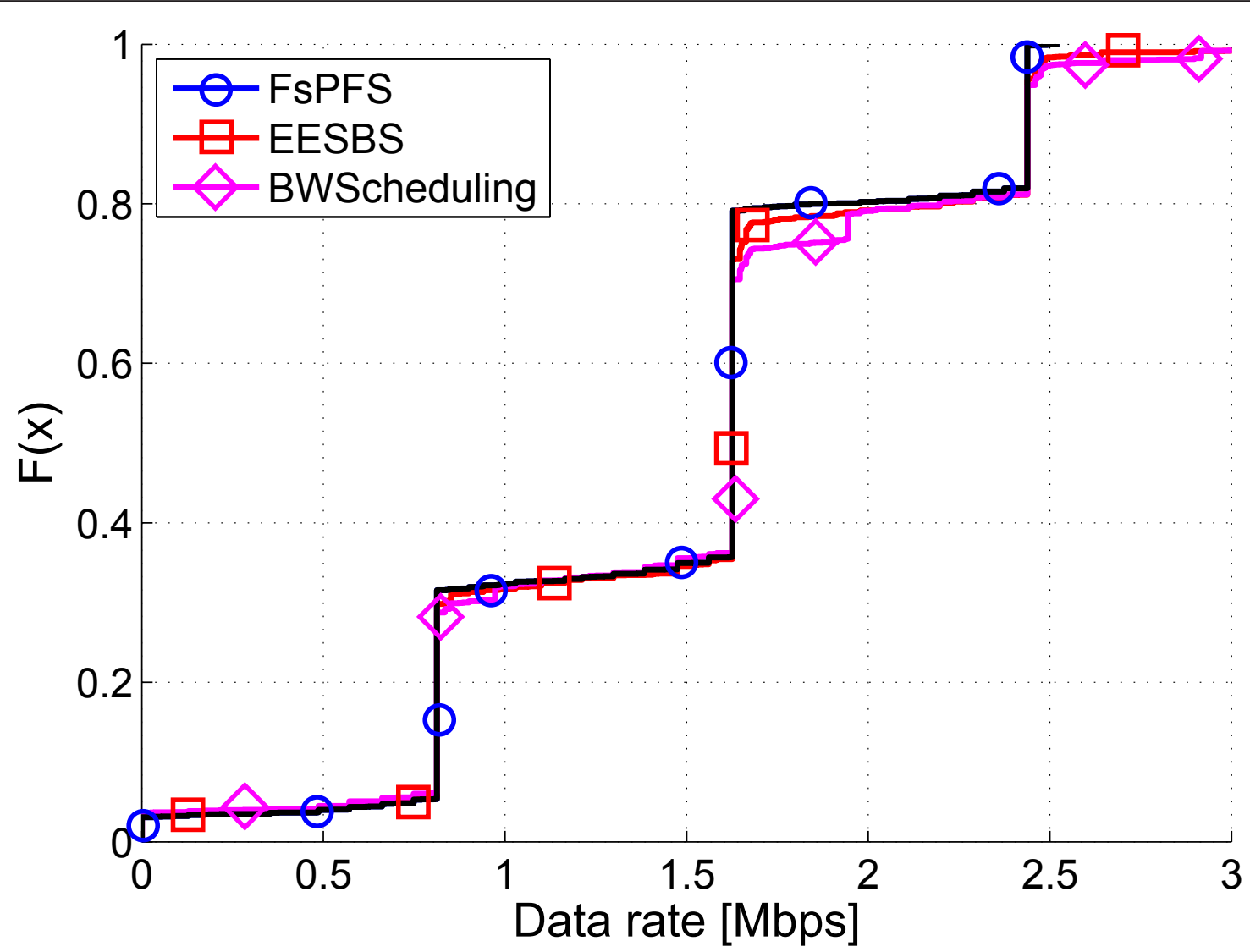

Figure 8 User data rate adjusted for $\phi=0.129$. All other parameters can be found in Table 1 . 
reasonable assumption since two independent studies have determined that LTE achieves link level spectral efficiency that is on average $2 \mathrm{~dB}$ away from the Shannon limit $[24,25]$. This guarantees that the values used are reasonable and close to the current state of the art.

The downlink transmission direction is simulated as it is currently the more energy intensive of the two. Data is collected from one TS after the system has settled to a stable resource allocation. Stability of resource allocation is important since it is desirable that RBs can be used by a user continuously, rather than be constantly contended for by a number of users. Channel condition changes are to be accounted for at the same time. If a more efficient allocation becomes available due to a change in channel conditions, it should be used instead. The theoretical concepts presented in this article can be applied in both the uplink and downlink transmission directions. However, the focus of this study is on downlink transmissions.

Two parameters are used to evaluate the proposed systems-ECG and data rate.

\subsection{Results}

In the context of this work, it is imperative to compare performance both in terms of data rate and energy efficiency.
Figure 8 presents the data rate performance of the systems. The simulation is set up so that whatever is achieved by the FsPF system is matched by the EESBS and bandwidth scheduling systems. This allows for a meaningful comparison when it comes to energy efficiency between the systems. Notable figures on the graph are $3 \%$ of the users experience outage, and the data rate per user is $1.6 \mathrm{Mbps}$ at the 50th percentile. The achieved data rates for the FsPF system do not match the target rates for the scenario since the simulated results are adjusted for overhead as well as being limited by the available modulation transmission modes.

In terms of ECG, the bandwidth scheduling system grants additional gains to EESBS. The results can be found in Figures 9 and 10. For the current and realistic future BS hardware models, the gains presented by the techniques are insignificant. However, for the ideal future BS model, there are significant energy reduction gains. EESBS and the combined system achieve energy reductions with respect to the benchmark of 29 and $38 \%$ at the 50th percentile respectively. The very high quiescent state drain with respect to the dynamic component of the BS energy consumption is responsible for the minimal energy gains observed for the current BS hardware model. At this point it is important to note

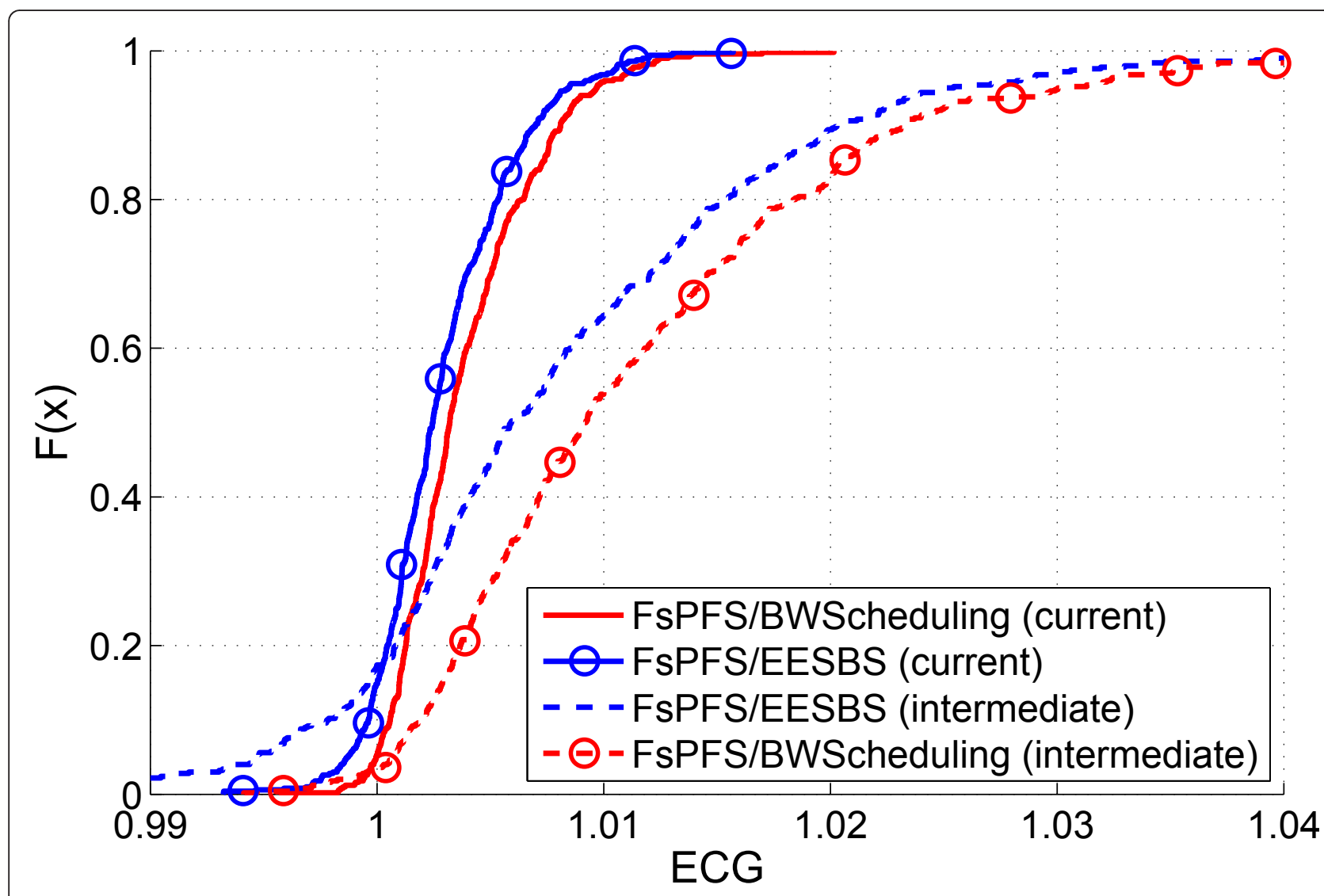

Figure 9 ECG for the current and realistic BS hardware models. All parameters can be found in Table 1. 


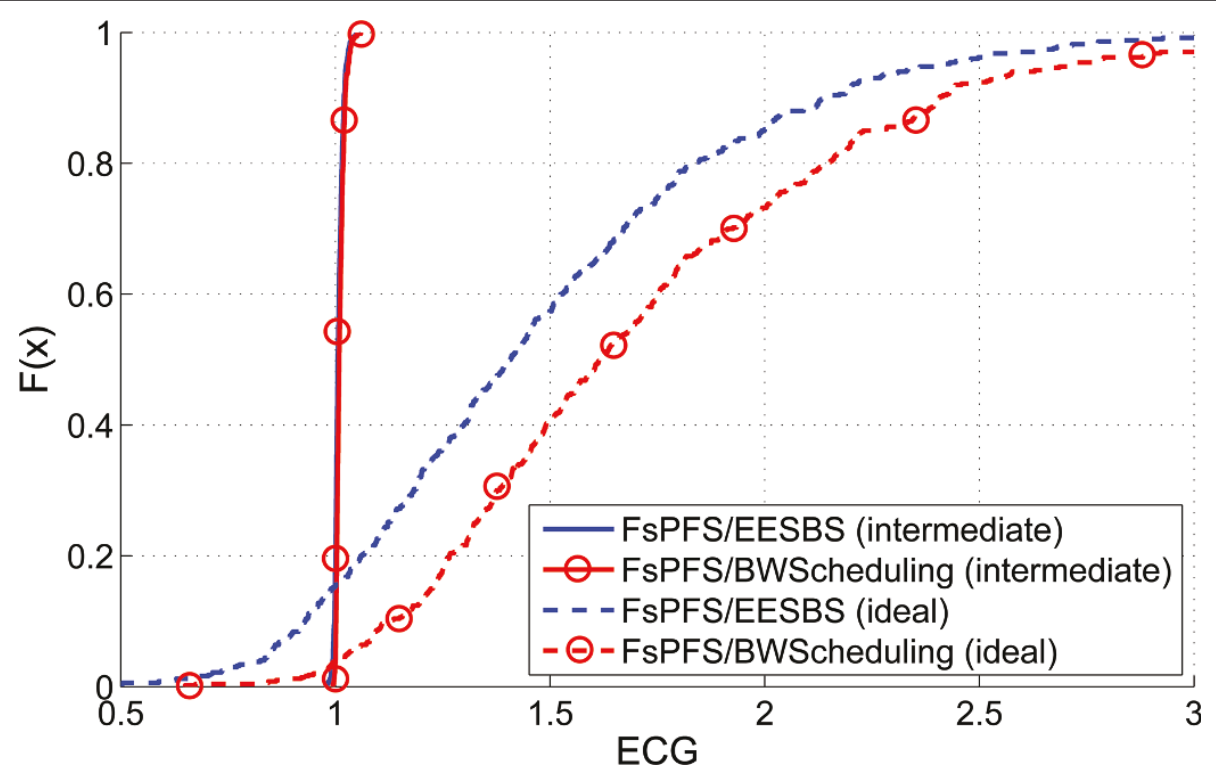

Figure 10 ECG for the realistic and ideal BS hardware models. All parameters can be found in Table 1 .

that the results indicate that the gains increase more than 3 -fold when the quiescent drain is halved-from 0.3 to $1 \%$. The combined system as expected achieves higher energy reduction over EESBS as it has more means of decreasing the energy consumption at its disposal. In practice, the real gain would lie between the results for the current and ideal models depending on the evolution of BS hardware in the future.

\section{Conclusion}

This article presents theoretical results on the performance of BEM in an environment that takes into account control channel overhead and variable energy efficiency in hardware. An expression for the ECG of BEM has been derived while taking into account BS hardware efficiency and control channel overhead. A complimentary technique, TCoM, is also introduced that relies on reduction of overhead to achieve energy gains. The new system, TCoM, is able to help achieve high gains in a realistic simulation scenario. The average reduction due to the combined bandwidth scheduling system for the simulated scenario is up to $38 \%$ depending on the BS hardware model. Realistic gains lie between the results presented for the current and ideal BS hardware models and their exact value would depend on the advances made in BS hardware.

\section{Acknowledgements}

The work reported in this article is part of the Green Radio Core Research Program of the Virtual Center of Excellence in Mobile \& Personal

Communications, Mobile VCE, http://www.mobilevce.com. This research has been funded by the industrial companies who are members of Mobile VCE. Prof. Haas acknowledges support from the Scottish Funding Council for his position in the Joint Research Institute in Signal and Image Processing, as part of the Edinburgh Research Partnership in Engineering and Mathematics, http://www.erp.ac.uk. We acknowledge support from the EPSRC under grant EP/G060584/1.

\section{Competing interests}

Parts of this work are subject to a patent application [26].

Received: 2 October 2011 Accepted: 28 May 2012

Published: 28 May 2012

\section{References}

1. Measuring the Information Society: The ICT Development Index. International Telecommunication Union, (2009)

2. C Han, T Harrold, S Armour, I Krikidis, S Videv, P Grant, H Haas, J Thompson, I Ku, C-X Wang, TA Le, M Nakhai, J Zhang, L Hanzo, Green radio: radio techniques to enable energy-efficient wireless networks. IEEE Commun Mag. 49(6), 46-54 (2011)

3. D Ferling, T Bohn, D Zeller, P Frenger, I Godor, Y Jading, W Tomaselli, Energy efficiency approaches for radio nodes, in Future Network and Mobile Summit, Florence, Italy, 1-9 (June 2010)

4. M Di Renzo, L Alonso, F Fitzek, A Foglar, F Granelli, F Graziosi, C Grueut, H Haas, G Kormentzas, A Perez, I Rodriguez, I Thompson, C Verikoukis, GREENET-An early stage training network in enabling technologies for green radio, in IEEE Vehicular Technology Conference (VTC Spring), Budapest, Hungary, 1-5 (15-18 May 2011)

5. A El Gamal, C Nair, B Prabhakar, E Uysal-Biyikoglu, S Zahedi, Energy-efficient scheduling of packet transmissions over wireless networks. in Proceedings of 21st Annual Joint Conference of the IEEE Computer and Communications Societies. 3, 1773-1782 (2002)

6. Y Yao, G Giannakis, Energy-efficient scheduling for wireless sensor networks. IEEE Trans Commun. 53(8), 1333-1342 (2005). doi:10.1109/ TCOMM.2005.852834

7. M Moretti, A Todini, A Baiocchi, G Dainelli, A layered architecture for fair resource allocation in multicellular multicarrier systems. IEEE Trans Veh Technol. 60(4), 1788-1798 (2011)

8. C Han, K Beh, M Nicolaou, S Armour, A Doufexi, Power efficient dynamic resource scheduling algorithms for LTE, in Vehicular Technology Conference Fall (VTC 2010-Fall), 2010 IEEE 72nd, Ottawa, Canada, 1-5 (Sept 2010)

9. B Ghimire, G Auer, H Haas, OFDMA-TDD networks with busy burst enabled grid-of-beam selection, in IEEE International Conference on Communications, Dresden, Germany, 1-6 (June 2009) 
10. H Zhang, H Dai, Cochannel interference mitigation and cooperative processing in downlink multicell multiuser MIMO networks. EURASIP J Wirel Commun Netw. 2004, 222-235 (2004). http://dx.doi.org/10.1155/ S1687147204406148

11. P Simoens, F Ali, B Vankeirsbilck, L Deboosere, F De Turck, B Dhoedt, P Demeester, R Torrea-Duran, Cross-layer optimization of radio sleep intervals to increase thin client energy efficiency. IEEE Commun Lett. 14(12), 1095-1097 (2010)

12. W Ye, J Heidemann, D Estrin, An energy-efficient MAC protocol for wireless sensor networks, in INFOCOM 2002. Twenty-First Annual Joint Conference of the IEEE Computer and Communications Societies Proceedings IEEE, New York, USA, pp. 3, 1567-1576 (2002)

13. K Han, S Choi, Performance analysis of sleep mode operation in IEEE 802.16e mobile broadband wireless access systems. in Vehicular Technology Conference, Melbourne. 3, 1141-1145 (Sept 2006)

14. R Wang, J Thompson, H Haas, A novel time-domain sleep mode design for energy-efficient LTE, in 4th International Symposium on Communications, Control and Signal Processing (ISCCSP), Limassol, 1-4 (Mar 2010)

15. F Richter, A Fehske, G Fettweis, Energy efficiency aspects of base station deployment strategies for cellular networks, in Vehicular Technology Conference Fall (NTC 2009-Fall), 2009 IEEE 70th, Anchorage, USA, 1-5 (Sept 2009)

16. T Bonald, A score-based opportunistic scheduler for fading radio channels, in Proc of the European Wireless Conference (EWC), Barcelona, Spain, 1-7 (24-27 Feb 2004)

17. GJ Foschini, Z Miljanic, A simple distributed autonomous power control algorithm and its convergence. IEEE Trans Veh Technol. 42(4), 641-646 (1993). doi:10.1109/25.260747

18. S Videv, H Haas, Energy-efficient scheduling and bandwidth-energy efficiency trade-off with low load, in Proc of the 2011 IEEE Internationa Communications Conference (ICC), Kyoto, Japan, 1-5 (5-9 June 2011)

19. S, Sesia, I Toufik, M Baker (eds.), LTE-The UMTS Long Term Evolution: From Theory to Practice, 1st edn. (Wiley, New York, 2009)

20. ICT-EARTH, D2.2, Energy Efficiency Analysis of the Reference Systems, Areas of Improvements and Target Breakdown, (Dec 2010) https://www.ict-earth. eu/publications/deliverables/deliverables.html. Accessed 7 Mar 2011

21. SB Lee, I Pefkianakis, A Meyerson, S Xu, S Lu, Proportional fair frequencydomain packet scheduling for 3GPP LTE uplink, in Proc of the IEEE International Conference on Computer Communications, Rio de Janeiro, Brazil, 2611-2615 (2009)

22. 3GPP, Further Advancements for E-UTRA Physical Layer Aspects (Release 9), 3GPP TR 36.814 V0.4.1 (2009-02), (Sept 2009) http://www.3gpp.org/ftp/ Specs/. Accessed 2 June 2009

23. IST-2003-507581 WINNER, D5.4 V1.0 Final Report on Link Level and System Level Channel Models, (Nov 2005) https://www.ist-winner.org/ DeliverableDocuments/. Accessed 15 Apr 2007

24. P Mogensen, W Na, I Kovacs, F Frederiksen, A Pokhariyal, K Pedersen, T Kolding, K Hugl, M Kuusela, LTE Capacity Compared to the Shannon Bound, in Proc IEEE 65th Vehicular Technology Conference Spring, Dublin, Ireland, 1234-1238 (Apr 2007)

25. C Mehlfuehrer, M Wrulich, JC Ikuno, D Bosanska, M Rupp, Simulating the long term evolution physical layer, in Proc 17th European Signal Processing Conference, Glasgow, Scotland, 1471-1478 (Aug 2009)

26. S Videv, H Haas, R Wang, JS Thompson, Operation of a Telecommunications System (under examination). Great Britain patent pub. no GB2482878 A, United States patent pub. no US2012/0044846 A1, priority date (Aug. 17, 2010)

doi:10.1186/1687-1499-2012-181

Cite this article as: Videv et al:: Resource allocation for energy efficient cellular systems. EURASIP Journal on Wireless Communications and Networking 2012 2012:181.

Submit your manuscript to a SpringerOpen ${ }^{\circ}$ journal and benefit from:

- Convenient online submission

- Rigorous peer review

- Immediate publication on acceptance

- Open access: articles freely available online

- High visibility within the field

- Retaining the copyright to your article

Submit your next manuscript at $\gg$ springeropen.com 